\title{
New inequalities for families without $k$ pairwise disjoint members
}

\author{
Peter Frankl, Andrey Kupavskii ${ }^{\dagger}$
}

\begin{abstract}
Some best possible inequalities are established for $k$-partition-free families (cf. Definition 11) and they are applied to prove a sharpening of a classical result of Kleitman concerning families without $k$ pairwise disjoint members.
\end{abstract}

\section{Introduction}

Let $n$ be a positive integer, $[n]=\{1,2, \ldots, n\}$ is the standard $n$-element set, $2^{[n]}$ its power set. For an integer $k \geq 2$ a family $\mathcal{F} \subset 2^{[n]}$ is called $k$-dependent if it contains no $k$ pairwise disjoint members. Similarly, if $\mathcal{F}_{1}, \ldots, \mathcal{F}_{k} \subset 2^{[n]}$ are not necessarily distinct families, we say that they are cross-dependent if there is no choice of $F_{i} \in \mathcal{F}_{i}, i=1, \ldots, k$, such that $F_{1}, \ldots, F_{k}$ are pairwise disjoint.

An important classical result of Kleitman [K] determines the maximal size, $|\mathcal{F}|$ of a $k$-dependent family $\mathcal{F} \subset 2^{[n]}$ for the cases $n \equiv-1$ or $0(\bmod k)$. In a recent paper [FK], Kupavskii and the author determined the maximum of $\left|\mathcal{F}_{1}\right|+\ldots+\left|\mathcal{F}_{k}\right|$ for cross-dependent families $\mathcal{F}_{i}$ for all values of $n \geq k \geq 3$. (Let us note that the easy case of $k=2$ was already solved by Erdős, Ko and Rado [EKR.)

*Rényi Institute, Budapest, Hungary

${ }^{\dagger}$ Moscow Institute of Physics and Technology, University of Birmingham; Email: kupavskii@yandex.ru Research supported by the grant RNF 16-11-10014 and by the EPSRC grant no. EP/N019504/1. 
Definition 1. For $k \geq 3$ and a family $\mathcal{F} \subset 2^{[n]}$ we say that $\mathcal{F}$ is $k$-partitionfree if $\mathcal{F}$ contains no $k$ pairwise disjoint members whose union is $[n]$.

Being $k$-partition-free is slightly less restrictive than being $k$-dependent.

For $0 \leq j \leq n$ let us use the notations $\mathcal{F}^{(j)}=\mathcal{F} \cap\left(\begin{array}{c}{[n]} \\ j\end{array}\right), f^{(j)}=\left|\mathcal{F}^{(j)}\right|$.

The following inequality is an important discovery of Kleitman [Kl].

Kleitman Lemma. Let $\mathcal{F} \subset 2^{[n]}$ be $k$-partition-free and let $j_{1}, j_{2}, \ldots, j_{k}$ be non-negative integers satisfying $j_{1}+\ldots+j_{k}=n$. Then

$$
\sum_{1 \leq i \leq k} \frac{f^{\left(j_{i}\right)}}{\left(\begin{array}{l}
n \\
j_{i}
\end{array}\right)} \leq k-1
$$

The proof of (1) is an easy averaging over all choices of pairwise disjoint sets $G_{1}, \ldots, G_{k}$ satisfying $\left|G_{i}\right|=j_{i}$ and noting that at least one of the relations $G_{i} \in \mathcal{F}$ fails.

Since the relation $j_{1}+\ldots+j_{k}=n$ is essential for proving (11) it is rather surprising that in certain cases one can prove the analogous inequality even if $j_{1}+\ldots+j_{k}>n$.

Let us first state our inequality for the case $k=3$.

Theorem 2. Let $m>\ell>0$ be integers, $n=3 m-\ell$. Suppose that $\mathcal{F} \subset 2^{[n]}$ is 3-partition-free. Then

$$
\frac{\left|\mathcal{F}^{(m-\ell)}\right|}{\left(\begin{array}{c}
n \\
m-\ell
\end{array}\right)}+\frac{\left|\mathcal{F}^{(m)}\right|}{\left(\begin{array}{c}
n \\
m
\end{array}\right)}+\frac{\left|\mathcal{F}^{(m+\ell)}\right|}{\left(\begin{array}{c}
n \\
m+\ell
\end{array}\right)} \leq 2 .
$$

Looking at the family $\left(\begin{array}{c}{[n]} \\ m\end{array}\right) \cup\left(\begin{array}{c}{[n]} \\ m+\ell\end{array}\right)$ shows that (2) is best possible.

To state our most general result let us say that the families $\mathcal{F}_{1}, \ldots, \mathcal{F}_{k} \subset$ $2^{[n]}$ are cross-partition-free if there is no choice of $F_{i} \in \mathcal{F}_{i}, i=1, \ldots, k$ such that $F_{1}, \ldots, F_{k}$ form a partition of $[n]$.

Theorem 3. Let $m>\ell>0$ be integers, $n=k m-\ell, k \geq 3$. For $1 \leq i \leq k$ let $\mathcal{F}_{i} \subset\left(\begin{array}{c}{[n]} \\ m-\ell\end{array}\right) \cup\left(\begin{array}{c}{[n]} \\ m\end{array}\right) \cup\left(\begin{array}{c}{[n]} \\ m+\ell\end{array}\right)$ and suppose that $\mathcal{F}_{1}, \ldots, \mathcal{F}_{k}$ are cross-partition-free. Then

$$
\sum_{1 \leq i \leq k} \frac{\left|\mathcal{F}_{i}^{(m-\ell)}\right|}{\left(\begin{array}{c}
n \\
m-\ell
\end{array}\right)}+\frac{\left|\mathcal{F}_{i}^{(m)}\right|}{\left(\begin{array}{c}
n \\
m
\end{array}\right)}+(k-2) \frac{\left|\mathcal{F}_{i}^{(m+\ell)}\right|}{\left(\begin{array}{c}
n \\
m+\ell
\end{array}\right)} \leq(k-1) k .
$$


Note that for $k=3$ and $\mathcal{F}_{1}=\ldots=\mathcal{F}_{k}$ the inequality (3) implies (2). The reason that we treat it separately is that both the statement and the proof are simple and hopefully give the reader the motivation to go through the more technical result (3).

The proofs of (22) and (3) are based on Katona's cyclic permutation method (cf. [Ka1], [Ka2]).

\section{The proof of (2)}

Let $x_{1}, x_{2}, \ldots, x_{3 m-\ell}, x_{1}$ be a random cyclic permutation of $\{1,2, \ldots, n\}$ (as indicated above, the element after $x_{n}$ is $\left.x_{1}\right)$. All $(n-1)$ ! cyclic permutations have the same probability $1 /(n-1)$ !. Set $d=(3 m-\ell, m)$.

We define three families, $\mathcal{B}, \mathcal{A}$ and $\mathcal{C} . \mathcal{B}=\left\{B_{1}, \ldots, B_{(3 m-\ell) / d}\right\}$ where $B_{r}=\left\{x_{j}:(r-1) m<j \leq r m\right\}, r=1, \ldots,(3 m-\ell) / d$. Note that the $B_{r}$ are arcs of $m$ consecutive elements $x_{j}$. Moreover, $(m, 3 m-\ell)=d$ guarantees that each of the $(3 m-\ell) / d \operatorname{arcs} B_{r}$ are distinct and the last element of $B_{n / d}$ is $x_{n}$.

Let us partition each $B_{r}$ as $B_{r}=A_{r} \cup D_{r}$ with $A_{r}$ being the arc consisting of the first $m-\ell$ elements. Formally, $A_{r}=\left\{x_{j}:(r-1) m<j \leq r m-\ell\right\}$, $D_{r}=B_{r} \backslash A_{r}$. Set $C_{r}=B_{r} \cup D_{r+1}$. Define

$$
\begin{aligned}
& \mathcal{A}=\left\{A_{r}: 1 \leq r \leq n / d\right\}, \\
& \mathcal{C}=\left\{C_{r}: 1 \leq r \leq n / d\right\} .
\end{aligned}
$$

Note that $C_{r}$ is not an arc but the union of two arcs and that it has the important property $C_{r} \cup A_{r+1}=B_{r} \cup B_{r+1}$ that we are going to use without further reference.

Lemma 2.1. If $\mathcal{F} \subset 2^{[n]}$ is 3-partition-free then

$$
|\mathcal{F} \cap \mathcal{A}|+|\mathcal{F} \cap \mathcal{B}|+|\mathcal{F} \cap \mathcal{C}| \leq 2 n / d .
$$

Proof of (2.1). Let $R=\left\{r: A_{r} \in \mathcal{F}\right\}, S=\left\{s: B_{s} \notin \mathcal{F}\right\}, T=\left\{t: C_{t} \notin \mathcal{F}\right\}$. (We consider $S$ and $T$ as sets on distinct ground sets.) To prove (2.1) it is sufficient to show

$$
|R| \leq|S|+|T| .
$$

We prove (2.2) by constructing an injection $\varphi$ from $R$ into $S \cup T$. 
First note that $B_{r}, B_{r+1}, A_{r+2}$ form a partition of $[n]$. This implies that if $r+2 \in R$ then at least one of $r, r+1$ is in $S$. If $r+1 \in S$, we set $\varphi(r+2)=r+1$. If not then we let provisionally $\varphi(r+2)=r$.

The only problem that might occur is that $r+1$ is also in $R$ and therefore $\varphi(r+2)=\varphi(r+1)=r$.

Noting that $C_{r}, A_{r+1}, A_{r+2}$ form a partition of $[n], r \in T$ follows. We change the value of $\varphi(r+2)$ to the element $r$ in $T$. This element is not allocated to any other $r^{\prime} \in R$ and the proof of (2.2) is complete.

To deduce (2) from (2.1) is easy averaging. For every $B \in \mathcal{B}$ the probability of $B \in \mathcal{F}$ is $\left|\mathcal{F}^{(m)}\right| /\left(\begin{array}{c}n \\ m\end{array}\right)$ by the uniform random choice of the permutation. The expected size $E(|\mathcal{F} \cap \mathcal{B}|)$ is

$$
|\mathcal{B}|\left|\mathcal{F}^{(m)}\right| /\left(\begin{array}{c}
n \\
m
\end{array}\right)=\frac{\left|\mathcal{F}^{(m)}\right|}{\left(\begin{array}{c}
n \\
m
\end{array}\right)} \cdot \frac{n}{d} .
$$

The same holds for $\mathcal{A}$ and $\mathcal{C}$ as well. By linearity of expectation and using the trivial fact that the expectation never exceeds the maximum, we infer

$\frac{n}{d}\left(\frac{\left|\mathcal{F}^{(m-\ell)}\right|}{\left(\begin{array}{c}n \\ m-\ell\end{array}\right)}+\frac{\left|\mathcal{F}^{(m)}\right|}{\left(\begin{array}{c}n \\ m\end{array}\right)}+\frac{\left|\mathcal{F}^{(m+\ell)}\right|}{\left(\begin{array}{c}n \\ m+\ell\end{array}\right)}\right)=E(|\mathcal{F} \cap \mathcal{A}|+|\mathcal{F} \cap \mathcal{B}|+|\mathcal{F} \cap \mathcal{C}|) \leq \frac{2 n}{d}$

Dividing by $\frac{n}{d}$ yields (2).

\section{The proof of (3)}

The proof is similar to that of (2) but both notationally and conceptually more complicated. Set $d=(k m-\ell, m)$ and $\bar{n}=n / d$. Fix a random cyclic permutation $x_{1}, \ldots, x_{n}$ of $\{1, \ldots, n\}$ and define again the $\bar{n}$ arcs of length $m, \mathcal{B}=\left\{B_{1}, \ldots, B_{\bar{n}}\right\}$ where $B_{r}=\left\{x_{q}:(r-1) m<q \leq r m\right\}$. The choice of $\bar{n}$ guarantees that $B_{\bar{n}}$ ends with the element $x_{n}$. This time we want to distribute these arcs among the $k$ families $\mathcal{F}_{i}, 1 \leq i \leq k$. For this reason let $b$ be the first positive integer such that $k$ divides $b \bar{n}$. Of course, $b=k /(\bar{n}, k)$.

We let $B_{r}^{(p)}$ be a copy of $B_{r}$ and make a circle of $b \bar{n}$ sets in the following order: $B_{1}^{(1)}, B_{2}^{(2)}, \ldots, B_{k}^{(k)}, B_{k+1}^{(1)}, \ldots, B_{b \bar{n}}^{(k)}$. For each pair $(r, p)$ we define the arc $A_{r}^{(p)} \subset B_{r}^{(p)}$ as the set of the first $m-\ell$ elements of $B_{r}^{(p)}$ and let $D_{r}^{(p)}$ be the rest: $D_{r}^{(p)}=B_{r}^{(p)} \backslash A_{r}^{(p)}$. For $1 \leq j \leq k-2$ we define the $(m+\ell)$-element sets

$$
C_{r}^{(p)}(j)=B_{r}^{(p)} \cup D_{r+j}^{(p+j)} \quad(r+j \text { is } \bmod \bar{n}, p+j \text { is } \bmod k) .
$$


Note that $C_{r}^{(p)}(j) \cup A_{r+j}^{(p+j)}=B_{r}^{(p)} \cup B_{r+j}^{(p+j)}$. For $1 \leq p \leq k$ let us define $\mathcal{B}^{(p)}=$ $\left\{B_{1}^{(p)}, \ldots, B_{\bar{n}}^{(p)}\right\}, \mathcal{A}^{(p)}=\left\{A_{1}^{(p)}, \ldots, A_{\bar{n}}^{(p)}\right\}$ and $\mathcal{C}_{j}^{(p)}=\left\{C_{r}^{(p)}(j): 1 \leq r \leq \bar{n}\right\}$, $1 \leq j \leq k-2$. Note that altogether we defined $(1+1+(k-2)) k=k^{2}$ families, each of size $b \bar{n} / k$. Therefore (3) will follow once we prove that out of these altogether $b \bar{n} k$ sets at most $b \bar{n}(k-1)$ are in the corresponding families $\mathcal{F}_{i}$. In other words we have to show that at least $b \bar{n}$ in total are missing.

Our plan is very simple. Fixing an arbitrary pair $(i, r), 1 \leq i \leq k$, $1 \leq r \leq \bar{n}$, we want to show that there is an integer, $0<t \leq k$ such that out of the following $t k$ sets at least $t$ are missing from the corresponding $\mathcal{F}_{i^{\prime}}$.

The list is $A_{r}^{(i)}, A_{r-1}^{(i-1)}, \ldots, A_{r-t+1}^{(i-t+1)} ; B_{r-1}^{(i-1)}, \ldots, B_{r-t}^{(i-t)} ; C_{r-1}^{(i-1)}(j), \ldots, C_{r-t}^{(i-t)}(j)$, $1 \leq j \leq k-2$.

To achieve this goal we prove a slightly stronger assertion. Since we do not need them for this statement, we remove the upper indices and let $C_{r}(j)$ denote the set $C_{r}^{(i)}(j)$ and the same with $B_{r}^{(i)}, A_{r}^{(i)}$.

Lemma 3.1. Let $r$ be fixed and consider the following $k$ groups of sets. $\mathcal{G}_{1}=\left\{A_{r}, B_{r-1}\right\}, \mathcal{G}_{2}=\left\{A_{r-1}, B_{r-2}, C_{r-2}(1)\right\}, \ldots, \mathcal{G}_{k-1}=\left\{A_{r-k+2}, B_{r-k+1}\right.$, $\left.C_{r-k+1}(1), \ldots, C_{r-k+1}(k-2)\right\}$. Suppose that we have families $\mathcal{H}_{i}, \mathcal{H}_{i} \subset \mathcal{G}_{i}$, $1 \leq i<k$ such that we cannot find $k$ members of $\mathcal{H}_{1} \cup \ldots \cup \mathcal{H}_{k-1}$ which partition $A_{r} \cup B_{r-1} \cup \ldots \cup B_{r-k+1}$. Then there exists $t, 1 \leq t \leq k$ satisfying

$$
\sum_{1 \leq s \leq t}\left|\mathcal{G}_{s} \backslash \mathcal{H}_{s}\right| \geq t
$$

Proof. First consider $\mathcal{H}_{1}$. If $\mathcal{H}_{1} \varsubsetneqq \mathcal{G}_{1}$ then (3.1) holds with $t=1$. If $\mathcal{H}_{1}=\mathcal{G}_{1}$ then the two members $A_{r}$ and $B_{r-1}$ partition $A_{r} \cup B_{r-1}$. Arguing indirectly, suppose that (3.1) does not hold and let $1 \leq t<k$ be the smallest integer such that $A_{r} \cup B_{r-1} \cup \ldots \cup B_{r-t}$ cannot be partitioned using the sets in $\mathcal{H}_{1} \cup \ldots \cup \mathcal{H}_{t}$.

By our assumptions $t$ exists and the above considerations show $t>1$ and $A_{r} \in \mathcal{H}_{1}$. The minimality of $t$ implies the existence of members $H_{i} \in \mathcal{H}_{i}$, $1 \leq i<t$ such that

$$
A_{r} \cup H_{1} \cup \ldots \cup H_{t-1}=A_{r} \cup B_{r-1} \cup \ldots \cup B_{r-(t-1)}
$$

is a partition with $H_{i} \in \mathcal{H}_{i}$. To conclude the proof we will prove that (3.1) holds for $t$.

First note that adding $B_{r-t}$ would make a partition of $A_{r} \cup B_{r-1} \cup \ldots \cup B_{r-t}$, implying $B_{r-t} \notin \mathcal{H}_{t}$. To exhibit $t-1$ further missing sets let us note the 
following important feature about the partition $H_{1} \cup \ldots \cup H_{t-1}=B_{r-1} \cup \ldots \cup$ $B_{r-(t-1)}$ : whenever a set $C_{r-s}(j)$ occurs it must come together with $A_{r-s+j}$ and the union of these two sets is $B_{r-s} \cup B_{r-s+j}$. Consequently, altering the order of the $H_{u}$, we can break up the partition as

$B_{r-1} \cup \ldots \cup B_{r-(t-1)}=B_{u_{1}} \cup \ldots \cup B_{u_{\ell}} \cup\left(B_{u_{\ell+1}} \cup B_{u_{\ell+2}}\right) \cup \ldots \cup\left(B_{u_{t-2}} \cup B_{u_{t-1}}\right)$.

To prove (3.1) we show the existence of distinct sets in $\bigcup_{1 \leq s \leq t} \mathcal{G}_{s} \backslash \mathcal{H}_{s}$, one for $B_{u_{i}}$ and two for $B_{u_{i}} \cup B_{u_{i+1}}$.

For $B_{u_{i}}$ note $C_{r-t}\left(u_{i}-r+t\right) \cup A_{u_{i}}=B_{r-t} \cup B_{u_{i}}$. Since $A_{r} \cup B_{r-1} \cup \ldots \cup B_{r-t}$ cannot be partitional by members of the $\mathcal{H}_{s}$, either $C_{r-t}\left(u_{i}-r+t\right)$ or $A_{u_{i}}$ is missing from the $\mathcal{H}_{s}$. For the case of $B_{v} \cup B_{w}$ (to simplify notation, $r-t<v<w \leq r-1)$ first note that one of the corresponding sets in $\mathcal{H}_{1} \cup \ldots \cup \mathcal{H}_{t-1}$ that partition $B_{v} \cup B_{w}$ is $A_{w}$ (the other is $C_{v}(w-v)$ ). Consider two partitions of $B_{r-t} \cup B_{v} \cup B_{w}$.

$$
\begin{aligned}
& C_{r-t}(w-r+t) \cup B_{v} \cup A_{w} \quad \text { and } \\
& C_{r-t}(v-r+t) \cup A_{v} \cup B_{w} .
\end{aligned}
$$

Since at least one set must be missing from both, we are done. Noting that the exhibited candidates for missing sets are all distinct, the proof of (3.1) is complete.

Equipped with (3.1) it is not hard to prove Lemma 3.1. Starting at an arbitrary $r$ we find, say, $t_{1}$ consecutive "groups" with at least a total of $t_{1}$ missing sets, $1 \leq t_{1} \leq k$. Then starting at $r-t_{1}$ we find $t_{2}$ such groups, etc. Going around the circle (of length $b \bar{n}$ ) the last position of the last group might not be $r+1$. However, since there are only $b \bar{n}$ members after making no more than $k$ full rounds we definitely have two sets of groups starting at the same element, say $r^{\prime}$. That is for the $t_{w}$ in between, say $t_{a}, t_{a+1}, \ldots, t_{a+q}$ one has $t_{a}+t_{a+1}+\ldots+t_{a+q}=c \cdot b \bar{n}$ with $c$ a positive integer. For these positions we exhibited altogether at least $c b \bar{n}$ missing sets and each of them is counted at most $c$ times. Therefore there are at least $b \bar{n}$ missing sets, proving Lemma 3.1.

Since Lemma 3.1 implies (3) by the same averaging argument as Lemma 2.1 implied (2), the proof of Theorem 3 is complete. 


\section{Applications}

Definition 4.1. For positive integers $n \geq k \geq 3$ let $p(n, k)$ denote the maximum of $|\mathcal{F}|$ over all $\mathcal{F} \subset 2^{[n]}$ that are $k$-partition-free.

\section{Theorem 4.2.}

$$
p(k m-1, k)=\sum_{j \geq m}\left(\begin{array}{c}
k m-1 \\
j
\end{array}\right)
$$

moreover the only $k$-partition-free family achieving equality in (4.1) is $\{G \subset$ $[k m-1]:|G| \geq m\}$.

Let us note that Kleitman [K] proved the same bound for the somewhat stronger restriction that the family is without $k$ pairwise disjoint sets. Also, Kleitman did not prove the uniqueness of the optimal family.

Proof. Since the case $m=1$ is trivial, we suppose $m \geq 2$. Our main tool is Theorem 3 applied with $\ell=1, \mathcal{F}_{1}=\ldots=\mathcal{F}_{k} \stackrel{\text { def }}{=} \mathcal{F}$. For the $k$-partition-free family $\mathcal{F} \subset 2^{[n]}, n=k m-1$ we get from (3)):

$$
\frac{\left|\mathcal{F}^{(m-1)}\right|}{\left(\begin{array}{c}
n \\
m-1
\end{array}\right)}+\frac{\left|\mathcal{F}^{(m)}\right|}{\left(\begin{array}{c}
n \\
m
\end{array}\right)}+(k-2) \frac{\left|\mathcal{F}^{(m+1)}\right|}{\left(\begin{array}{c}
n \\
m+1
\end{array}\right)} \leq k-1 .
$$

Setting $y(j)=\left(\begin{array}{c}n \\ j\end{array}\right)-\left|\mathcal{F}^{(j)}\right|$ we obtain

$$
\frac{y(m-1)}{\left(\begin{array}{c}
n \\
m-1
\end{array}\right)}+\frac{y(m)}{\left(\begin{array}{c}
n \\
m
\end{array}\right)}+(k-2) \frac{y(m+1)}{\left(\begin{array}{c}
n \\
m+1
\end{array}\right)} \geq 1 .
$$

Note $\left(\begin{array}{c}k m-1 \\ m\end{array}\right)=(k-1)\left(\begin{array}{c}k m-1 \\ m-1\end{array}\right)$ and for further use

$$
\left(\begin{array}{c}
k m-1 \\
m-j+1
\end{array}\right) /\left(\begin{array}{c}
k m-1 \\
m-j
\end{array}\right)=\frac{(k-1) m+j-1}{m-j+1}>k-1 \text { for } j \geq 2 .
$$

Using $\left(\begin{array}{c}k m-1 \\ m+i\end{array}\right) \geq\left(\begin{array}{c}k m-1 \\ m\end{array}\right)$ (valid for $i<m$ ) (4.2) yields the following inequality.

$$
y(m-1)+\frac{1}{k-1} y(m)+\frac{k-2}{k-1} y(m+1) \geq\left(\begin{array}{c}
k m-1 \\
m-1
\end{array}\right) .
$$

Let us apply (11) with

$$
\left(j_{1}, \ldots, j_{k}\right)=(m-\ell, m, m, \ldots, m, m+\ell-1) \text { for } \ell=2,3, \ldots, m \text {. }
$$


Multiplying both sides by $\left(\begin{array}{c}n \\ m-\ell\end{array}\right)$ we obtain

$$
y(m-\ell)+\frac{(k-2)}{(k-1)^{\ell}} y(m)+\frac{1}{(k-1)^{\ell}} y(m+\ell-1) \geq\left(\begin{array}{c}
k m-1 \\
m-\ell
\end{array}\right)
$$

(we used (4.3) and $\left(\begin{array}{c}n \\ m+\ell-1\end{array}\right)>\left(\begin{array}{c}n \\ m\end{array}\right)$ ). We want to add (4.4) and the sum of (4.5) over $2 \leq \ell \leq m$. For $\ell>2$ the term $y(m+\ell-1)$ occurs only once and its coefficient is smaller than $\frac{1}{k-1}<1$. The term $y(m+1)$ has coefficient

$$
\frac{k-2}{k-1}+\frac{1}{(k-1)^{2}}<\frac{k-2}{k-1}+\frac{1}{k-1}=1 \text { also. }
$$

Finally $(k-1)^{-2}+(k-1)^{-3}+\ldots=\frac{k-1}{k-2} \cdot \frac{1}{(k-1)^{2}}=\frac{1}{(k-2)} \cdot \frac{1}{k-1}$. Thus the total coefficient of $y(m)$ will be less than $\frac{2}{k-1} \leq 1$. That is, we obtain an inequality of the form

$$
y(0)+y(1)+\ldots+y(m-1)+c_{m} y(m)+\ldots+c_{2 m} y(2 m) \geq \sum_{0 \leq j<m}\left(\begin{array}{l}
n \\
j
\end{array}\right)
$$

with $c(m+i)<1$ for $0 \leq i \leq m$. Consequently, $|\mathcal{F}| \leq 2^{n}-\sum_{0 \leq j<m}\left(\begin{array}{l}n \\ j\end{array}\right)=$ $\sum_{j \geq m}\left(\begin{array}{c}n \\ m\end{array}\right)$, as desired. Moreover, in case of equality, $y(m+i)=0$ must hold because of $c_{m+i}<1$ for all $0 \leq i \leq m$. Plugging these values into (4.4) and (4.5), $y(m-\ell)=\left(\begin{array}{c}n \\ m-\ell\end{array}\right)$ follows for all $1 \leq \ell \leq m$. That is, $\mathcal{F}=\{F \subset[n]$ : $|F| \geq m\}$ concluding the proof of the uniqueness.

Remark. If we used (11) instead of Theorem 3 then instead of (4.4) we would have

$$
y(m-1)+y(m) \geq\left(\begin{array}{c}
k m-1 \\
m
\end{array}\right) .
$$

Thus adding more equalities to it would make the coefficient of $y(m)$ greater than 1.

Definition 4.3. The not necessarily distinct families $\mathcal{F}_{1}, \ldots, \mathcal{F}_{k}$ are called cross-dependent if there is no choice of $F_{1} \in \mathcal{F}_{1}, \ldots, F_{k} \in \mathcal{F}_{k}$ that are pairwise disjoint.

Let us recall the following recent result of Kupavskii and the author. 
Theorem $4.4([\mathrm{FK}])$. Suppose that $\mathcal{F}_{1}, \ldots, \mathcal{F}_{k} \subset 2^{[n]}, n=m k-1$, are cross-dependent. Then one has:

$$
\left|\mathcal{F}_{1}\right|+\ldots+\left|\mathcal{F}_{k}\right| \leq k \cdot \sum_{j \geq m}\left(\begin{array}{c}
m k-1 \\
j
\end{array}\right) .
$$

One can use Theorem 3 to prove (4.6) under the weaker assumption of being cross-partition-free and show that equality holds only if

$$
\mathcal{F}_{1}=\ldots=\mathcal{F}_{k}=\{F \subset[n]:|F| \geq m\} .
$$

We leave the details to the interested reader.

Let us mention that in [FK] the maximum of $\left|\mathcal{F}_{1}\right|+\ldots+\left|\mathcal{F}_{k}\right|$ is determined for all values of $n$ and $k$. The methods presented in this paper seem to be insufficient to tackle the cases $n \not \equiv-1(\bmod k)$.

\section{References}

[EKR] P. Erdős, C. Ko, R. Rado, Intersection theorems for systems of finite sets, Quart. J. Math. Oxford Second Series 12 (1961), 313-320.

[FK] P. Frankl, A. Kupavskii, Two problems of P. Erdős on matchings in set families, submitted, arXiv:1607.06126

[Ka1] G. O. H. Katona, A simple proof of the Erdős-Chao Ko-Rado theorem, J. Combin. Theory Ser. B 13 (1972), 183-184.

[Ka2] G. O. H. Katona, The cycle method and its limits, in: Numbers, Information and Complexity (I. Althöfer, Ning Cai, G. Dueck, L. Khachatrian, M.S. Pinsker, A. Sárközy, I. Wegener, Zhen Zhang, eds.), Kluwer, 2000, pp. 129-141.

[Kl] D. J. Kleitman, Maximal number of subsets of a finite set no $k$ of which are pairwise disjoint, J. Combinatorial Th. 5 (1968), 157-163. 